This item was submitted to Loughborough's Research Repository by the author.

Items in Figshare are protected by copyright, with all rights reserved, unless otherwise indicated.

\title{
Motion of a pair of gravitating bodies in dark energy presence: small deviations from Keplerian motion
}

PLEASE CITE THE PUBLISHED VERSION

https://doi.org/10.1134/S1063772920100054

\section{PUBLISHER}

Springer Verlag

VERSION

AM (Accepted Manuscript)

\section{PUBLISHER STATEMENT}

This is a post-peer-review, pre-copyedit version of an article published in Astronomy Reports. The final authenticated version is available online at: https://doi.org/10.1134/S1063772920100054

\section{LICENCE}

CC BY-NC-ND 4.0

\section{REPOSITORY RECORD}

Neishtadt, Anatoly, and Gennady Bisnovatyi-Kogan. 2020. "Motion of a Pair of Gravitating Bodies in Dark Energy Presence: Small Deviations from Keplerian Motion”. Loughborough University. https://hdl.handle.net/2134/12816362.v1. 


\title{
Движение пары гравитирующих тел в присутствии темной энергии: малые отклонения от кеплеровского
} движения

\author{
Нейштадт А.И. ${ }^{1,2}$, Бисноватый-Коган Г.С. ${ }^{1 *}$
}

\begin{abstract}
Аннотация
Исследована задача о движении двух гравитирующих тел при наличии ТЭ, рассматриваемой как возмущающий фактор. В дополнение к частоте прецессии орбиты, полученной в предыдущих работах, вычисляется поправка к частоте орбитального движения за счет ТЭ. Эта поправка имеет тот же порядок, что и частота вращения перицентра, и потребовала более точного рассмотрения по сравнению с предыдущими. На основе используемого метода можно получить следующие по порядку приближения и исследовать отличия от усредненного движения.
\end{abstract}

\section{1 Введение}

Открытие темной энергии (ТЭ) в современной вселенной было сделано на основе наблюдения сверхновых типа Іа при красных смещениях $z \leq 1[1,2]$, и измерения спектра флуктуаций космического микроволнового фонового излучения (KMФ) [3,4]. Эти наблюдения привели к определению величины космологической постоянной $\Lambda \approx 10^{-56} \mathrm{~cm}^{-2}$, отождествленной с ТЭ. Влияние ТЭ на свойства космологического расширения в современную эпоху рассматривалось в обзорах [5] - [12].

В более раннюю эпоху наблюдательные ограничения на величину космологической постоянной $\Lambda$ и плотности темной энергии $\rho_{D E}=\frac{\Lambda c^{2}}{8 \pi G}$ были получены на основе прецизионных наблюдений тайминга двойных радиопульсаров и движения планет Солнечной системы. Анализ данных о прецессии перигелия Меркурия для получения ограничений на величину $\Lambda$ проводился различными авторами [13], [14], [15], получавшими сильно различающиеся

\footnotetext{
${ }^{* 1}$ Институт космических исследований РАН, Москва. Адрес эл. почты: aneishta@iki.rssi.ru, gkogan@iki.rssi.ru . ${ }^{2}$ Университет Лафборо, Великобритания.
} 
значения этих ограничений. В работе [15] был получен верхний предел $\Lambda<4 \cdot 10^{-45} \mathrm{~cm}^{-2}$, упомянутый в последующих работах [16] - [19]. Таким образом, измеренное значение $\Lambda$ оказалось более чем на 10 порядков меньше этого верхнего предела.

Гравитомагнитный эффект хода часов при их движении по орбите вокруг вращающегося тела состоит в разности скорости хода часов при их вращении по противоположно направленным орбитам [20,21]. Влияние космологической постоянной на этот эффект, а также влияние $\Lambda$ на релятивистскую прецессию перигелия, исследовались в работе [22]. Влияние космологической постоянной на прецессию перигелия Земли и Марса, исследованное в работах [15] и [24], привело к ограничению на значение $\Lambda$ в виде $\Lambda<1 \cdot 10^{-46} \mathrm{~cm}^{-2}$. Различные релятивистские эффекты в Солнечной системе на основе метрики Шварцшильда - де Ситтера были рассмотрены в работе [25]. Влияние малой дополнительной центрально-симметричной силы и космологического расширения вселенной на движение по кеплеровским орбитам исследовалось в работах [26] - [29].

В работах [30] - [32] было показано, что влияние ТЭ на динамику во внешних областях скоплений галактик может быть достаточно сильным. При рассмотрении относительного движения двух богатых скоплений влияние космологической постоянной может быть определяющим [33]. Оценка влияния измеренной космологической постоянной $\Lambda \approx 10^{-56}$ $\mathrm{cm}^{-2}$ на прецессию орбиты планет вокруг Солнца сделана в [22]. Было показано, что для солнечной системы это влияние очень мало, и частота прецессии орбиты Земли составляет величину $\sim 10^{-14}$ от эйнштейновского значения. В [22] показано, что отношение этих частот пропорционально 4-й степени размера большей полуоси орбиты $\sim a^{4}$, т.е. зависимость от периода обращения $\sim T^{8 / 3}$. Т.о. для Меркурия эта величина меньше в $\left(T_{\oplus} / T_{\text {Mercury }}\right)^{8 / 3} \approx 365 / 88 \approx 44$ раза. Относительное влияние ТЭ на частоту прецессии уменьшается с приближением орбиты планеты к Солнцу, т.к. роль гравитации Солнца растет, а вклад ТЭ падает, ввиду постоянной плотности ее энергии. Гораздо сильнее влияние ТЭ на относительное движение галактик, когда роль ТЭ может стать сравнимой с притяжением между галактиками [30-32]. Временные масштабы при этом столь велики, что наблюдать можно только результаты длительного действия различных факторов.

Решение задачи Кеплера в присутствии ТЭ было получено в квази-ньютоновском приближении в работе [34]. Общее аналитическое решение, справедливое для произвольных $\Lambda$, было записано через различные эллиптические интегралы с использованием табличных формул [35]. Это громоздкое решение не очень удобно для анализа различных физических эффектов в задаче Кеплера, связанных с наличием ТЭ. В настоящей работе, в задаче о движении двух гравитирующих тел, наличие ТЭ рассматривается как возмущающий фактор, аналогично [22]. При этом используется несколько отличающийся метод усреднения по траектории. В дополнение к частоте прецессии орбиты, полученной в [22], вычисляется поправка к частоте орбитального движения (или, эквивалентно, к интервалу времени 
между двумя прохождениями через перицентр) за счет ТЭ. Эта поправка имеет тот же порядок, что и частота вращения перицентра, и потребовала более точного рассмотрения по сравнению с [22]. На основе используемого метода можно получить следующие по порядку приближения и исследовать отличия от усредненного движения.

\section{2 Кеплеровское движение в вакууме}

Движение двух гравитирующих тел с массами $m_{1}, m_{2}$ относительно друг друга сводится уравнению Кеплера, которое описывает движение тела с приведённой массой $m=\frac{m_{1} m_{2}}{m_{1}+m_{2}}$ вокруг неподвижного тела массы $M=m_{1}+m_{2}$. Система уравнений для компонент $(r, \phi)$ вектора расстояния $\mathbf{r}$ между гравитирующими телами $m$ и $M$ имеет интегралы энергии $E$ и углового момента $L[36]$

$$
E=\frac{m v^{2}}{2}+\frac{L^{2}}{2 m r^{2}}-\frac{G M m}{r}, \quad L=m v_{\phi} r, \quad v=\frac{d r}{d t}, \quad v_{\phi}=r \frac{d \phi}{d t} .
$$

Решение этой системы при $E<0$ описывает замкнутые эллиптические траектории вида [36]

$$
r=\frac{a\left(1-\epsilon^{2}\right)}{1+\epsilon \cos (\phi-g)} .
$$

Большая полуось $a$ и эксцентриситет $\epsilon$ эллипса равны

$$
a=\frac{G M}{2|E|}, \quad \epsilon=\sqrt{1+\frac{2 E L^{2}}{m^{3}(G M)^{2}}},
$$

долгота перицентра $g$ определяет направление линии апсид, истинная аномалия $\phi-g$ определяет положение точки на орбите ( $\phi$ - долгота).

\section{3 Прецессия орбит в присутствии ТЭ}

Присутствие темной энергии в квази-ньютоновском приближении учитывается дополнительной радиальной силой отталкивания, что приводит к изменению интеграла энергии, который принимает следующий вид

$$
E=\frac{m v^{2}}{2}+\frac{L^{2}}{2 m r^{2}}-\frac{G M m}{r}-\frac{\Lambda m c^{2}}{6} r^{2}
$$

Как и во всяком центральном поле, движение происходит в плоскости. Орбиты в этой плоскости становятся незамкнутыми. Если влияние темной энергии считать малым возмущающим фактором, то орбита может быть представлена в виде медленно прецессирующей кеплеровской орбиты. В [22] скорости изменения уравнения оскулирующих элементов в 
этой задаче усреднены по периоду кеплеровского движения. В результате получены следующие усредненные уравнения

$$
\left\langle\frac{d a}{d t}\right\rangle=0, \quad\left\langle\frac{d \epsilon}{d t}\right\rangle=0, \quad\left\langle\frac{d g}{d t}\right\rangle=\frac{\Lambda c^{2}}{2 \omega} \sqrt{1-\epsilon^{2}} .
$$

Последнее соотношение в (3.2) дает частоту прецессии перицентра.

\section{4 Уравнения движения в гамильтоновой форме}

Движение происходит в плоскости и описывается гамильтоновой системой с двумя степенями свободы. Будем использовать канонические переменные Делоне $I_{1}, I_{2}, \varphi_{1}, \varphi_{2}$ ( [37], стр.340):

$$
\begin{aligned}
& I_{1}=\sqrt{\mu a}, \quad \varphi_{1}=l, \\
& I_{2}=\sqrt{\mu a\left(1-\epsilon^{2}\right)}, \quad \varphi_{2}=g .
\end{aligned}
$$

Здесь $\mu=G M$, a $l$ - средняя аномалия в задаче Кеплера. Напомним, что в задаче Кеплера средняя аномалия - это угловая переменная, которая равномерно изменяется при движении тел по орбите. Скорость ее изменения (среднее движение) $\omega=\sqrt{G M} / a^{3 / 2}$. Средняя аномалия выражается через эксцентрическую аномалию $\xi$ с помощью уравнения Кеплеpa $[36,37]$

$$
l=\xi-\epsilon \sin \xi
$$

Гамильтониан задачи $H$ пропорционален полной энергии, $H=E / m$. В переменных Делоне гамильтониан записывается в виде

$$
\begin{aligned}
H & =H_{0}\left(I_{1}\right)+\varepsilon H_{1}\left(I_{1}, I_{2}, \varphi_{1}\right), \\
H_{0} & =-\frac{\mu^{2}}{2 I_{1}^{2}}, \quad H_{1}=-r^{2} .
\end{aligned}
$$

Здесь $r^{2}$ должно быть выражено через $I_{1}, I_{2}, \varphi_{1}$. Величина $\varepsilon=\frac{\Lambda c^{2}}{6}$ будет считаться малым параметром задачи, по которому проводятся разложения (это не безразмерная величина, но ее удобно использовать в разложениях, чтобы не вводить дополнительно безразмерный малый параметр). Этому гамильтониану соответствуют уравнения движения

$$
\begin{aligned}
& \dot{I}_{1}=-\varepsilon \frac{\partial H_{1}}{\partial \varphi_{1}}, \quad \dot{\varphi}_{1}=\frac{\partial H_{0}}{\partial I_{1}}+\varepsilon \frac{\partial H_{1}}{\partial I_{1}}, \\
& \dot{I}_{2}=-\varepsilon \frac{\partial H_{1}}{\partial \varphi_{2}}=0, \quad \dot{\varphi}_{2}=\varepsilon \frac{\partial H_{1}}{\partial I_{2}} .
\end{aligned}
$$


В этой записи учтено, что $H_{0}$ зависит только от $I_{1}$, и поэтому частные производные $H_{0}$ по остальным переменным обращаются в 0.

Величина $I_{2}$ пропорциональна угловому моменту, $I_{2}=L / m$, и является первым интегралом задачи, $I_{2}=$ const. В терминах уравнений (4.4) это связано с тем, что гамильтониан не зависит от угла $\varphi_{2}$. Соответственно, частная производная гамильтониана по $\varphi_{2}$ тождественно обращается в 0 , а канонически сопряженная к $\varphi_{2}$ переменная $I_{2}$ является первым интегралом.

Задача имеет также интеграл энергии $H=H_{0}\left(I_{1}\right)+\varepsilon H_{1}\left(I_{1}, I_{2}, \varphi_{1}\right)=h=$ const, так как гамильтониан не зависит явно от времени.

Рассматриваемая задача интегрируема: для $I_{1}$ и $\varphi_{1}$ получается гамильтонова система с одной степенью свободы, зависящая от $I_{2}$ как от параметра. По найденным решениям этой системы можно затем получить зависимость $\varphi_{2}$ от времени квадратурой [34].

\section{5 Применение канонической теории возмущений}

\section{1 Общее описание процедуры Делоне-Цейпеля}

Мы будем строить приближенные решения при малых $\varepsilon$. В рассматриваемой задаче одна быстро меняющаяся угловая переменная, угол $\varphi_{1}$, и две медленно меняющиеся переменные, $I_{1}$ и $\varphi_{2}$ (переменную $I_{2}$ можно рассматривать как параметр задачи). Для описания динамики будем использовать процедуру канонической теории возмущений в форме Делоне-Цейпеля [37], стр. 426. ' Эта процедура применительно к рассматриваемой задаче состоит в том, что делается каноническая $2 \pi$-периодическая по $\varphi_{1}, \varphi_{2}$, близкая к тождественной (отличающаяся от тождественной величинами порядка $\varepsilon$ ) замена переменных $I_{1}, I_{2}, \varphi_{1}, \varphi_{2} \mapsto J_{1}, J_{2}, \psi_{1}, \psi_{2}$, такая, что гамильтониан в новых переменных зависит от быстрой угловой переменной $\psi_{1}$ лишь в членах выше заданного порядка. Если мы хотим исключить $\psi_{1}$ из гамильтониана вплоть до членов порядка $\varepsilon^{n}$ включительно в разложении по малому параметру $\varepsilon$, то производящая функция нужной замены переменных ищется в виде многочлена степени $n$ по $\varepsilon$. Отбрасывая в гамильтониане малый член порядка $\varepsilon^{n+1}$, получаем гамильтониан, не зависящий от переменной $\psi_{1}$. В полученной гамильтоновой системе величина $J_{1}$ является первым интегралом, $J_{1}=$ const, в соответствии с гамильтоновыми уравнениями движения. Для остальных переменных получается система с меньшим на еди-

\footnotetext{
аЭта процедура была предложены для неинтегрируемых задач, но она полезна и при построении приближенных решений в более простых случаях, когда рассматриваемая задача интегрируема и имеет точное решение. K тем же теоретическим результатам привело бы и использоание более приспособленой для компьютерной реализации процедуры Хори-Депри [38]. В первом приближении процедуры Делоне-Цейпеля и Хори-Депри эквивалентны.
} 
ницу числом степеней свободы, зависящая от $J_{1}$ как от параметра, причем все переменные в этой системе меняются медленно. После решения этой системы приближенное решение исходной системы получается с помощью обращения построенной замены переменных.

Для рассматриваемой задачи описанная процедура еще упрощается из-за того, что гамильтониан не зависит от $\varphi_{2}$. Тогда процедура организуется так, что новый гамильтониан не зависит от $\psi_{2}$, и $J_{2}=I_{2}=$ const. Получается, что величины $\dot{\psi}_{1}, \dot{\psi}_{2}$ постоянны. Это средние частоты изменения углов $\varphi_{1}, \varphi_{2}$.

Мы ограничимся первым приближением описанной процедуры: возьмем $n=1$. Делаем каноническую замену переменных $\left(I_{1}, I_{2}, \varphi_{1}, \varphi_{2}\right) \mapsto\left(J_{1}, J_{2}, \psi_{1}, \psi_{2}\right)$ с производящей функцией

$$
W=J_{1} \varphi_{1}+J_{2} \varphi_{2}+\varepsilon S\left(J_{1}, J_{2}, \varphi_{1}\right),
$$

где $S$ - пока не определенная $2 \pi$-периодическая по $\varphi_{1}$ функция (производящая функция зависит от старых координат $\varphi_{1}, \varphi_{2}$ и новых импульсов $\left.J_{1}, J_{2}\right)$.

Старые и новые переменные связаны соотношениями

$$
I_{1}=J_{1}+\varepsilon \frac{\partial S}{\partial \varphi_{1}}, I_{2}=J_{2}+\varepsilon \frac{\partial S}{\partial \varphi_{2}}=J_{2}, \psi_{1}=\varphi_{1}+\varepsilon \frac{\partial S}{\partial J_{1}}, \psi_{2}=\varphi_{1}+\varepsilon \frac{\partial S}{\partial J_{2}} .
$$

Замена переменных (5.1) близка к тождественной.

Подставляя в гамильтониан выражение для $I_{1}$ через $J_{1}, \varphi_{1}, I_{2}$ из $(5.1)$, получаем

$$
\begin{aligned}
H & =H_{0}\left(J_{1}+\varepsilon \frac{\partial S}{\partial \varphi_{1}}\right)+\varepsilon H_{1}\left(J_{1}+\varepsilon \frac{\partial S}{\partial \varphi_{1}}, I_{2}, \varphi_{1}\right) \\
& =H_{0}\left(J_{1}\right)+\varepsilon\left[\frac{\partial H_{0}\left(J_{1}\right)}{\partial J_{1}} \frac{\partial S}{\partial \varphi_{1}}+H_{1}\left(J_{1}, I_{2}, \varphi_{1}\right)\right]+O\left(\varepsilon^{2}\right) .
\end{aligned}
$$

Обозначим $\bar{H}_{1}$ среднее от $H_{1}$ по $\varphi_{1}$. Хотим выбрать $S$ так, чтобы было

$$
\frac{\partial H_{0}\left(J_{1}\right)}{\partial J_{1}} \frac{\partial S}{\partial \varphi_{1}}+H_{1}\left(J_{1}, I_{2}, \varphi_{1}\right)=\bar{H}_{1}\left(J_{1}, I_{2}\right) .
$$

Тогда $S$ получается интегрированием по $\varphi_{1}$ из соотношения

$$
\frac{\partial S}{\partial \varphi_{1}}=-\frac{1}{\partial H_{0}\left(J_{1}\right) / \partial J_{1}}\left[H_{1}\left(J_{1}, I_{2}, \varphi_{1}\right)-\bar{H}_{1}\left(J_{1}, I_{2}\right)\right] .
$$

Так как правая часть (5.2) $2 \pi$-периодическая функция от $\varphi_{1}$ со средним 0 , то $S$ получается $2 \pi$-периодической функцией от $\varphi_{1}$. Мы будем выбирать функцию $S$ так, что ее среднее по $\varphi_{1}$ равно 0. Гамильтониан в новых переменных

$$
H=H_{0}\left(J_{1}\right)+\varepsilon \bar{H}_{1}\left(J_{1}, I_{2}\right)+O\left(\varepsilon^{2}\right) .
$$

Пренебрегая членом порядка $\varepsilon^{2}$, получаем гамильтониан $\mathcal{H}=H_{0}\left(J_{1}\right)+\varepsilon \bar{H}_{1}\left(J_{1}, I_{2}\right)$ и уравнения движения

$$
\begin{aligned}
\dot{J}_{1} & =0, \quad \dot{I}_{2}=0 \\
\dot{\psi}_{1} & =\frac{\partial H_{0}\left(J_{1}\right)}{\partial J_{1}}+\varepsilon \frac{\partial \bar{H}_{1}}{\partial J_{1}}, \quad \dot{\psi}_{2}=\varepsilon \frac{\partial \bar{H}_{1}}{\partial I_{2}} .
\end{aligned}
$$


Здесь $\bar{H}_{1}=\bar{H}_{1}\left(J_{1}, I_{2}\right)$.

\section{2 Поправки к кеплеровскому движению}

Проведем выкладки.

Среднее от $H_{1}$ по $\varphi_{1}$ есть

$$
\bar{H}_{1}=\frac{1}{2 \pi} \int_{0}^{2 \pi} H_{1} d \varphi_{1}=-\frac{1}{2 \pi} \int_{0}^{2 \pi} r^{2} d \varphi_{1},
$$

т.е., с точностью до знака, $\bar{H}_{1}$ - это нулевой член в известном разложении функции $r^{2}$ в ряд Фурье по средней аномалии (см., например, [37], с. 236, формула (2.3.8)):

$$
\bar{H}_{1}=-a^{2}\left(1+\frac{3 \epsilon^{2}}{2}\right) \text {. }
$$

Поскольку

$$
a^{2}=I_{1}^{4} / \mu^{2}, \epsilon^{2}=1-\frac{I_{2}^{2}}{I_{1}^{2}},
$$

получаем

$$
\bar{H}_{1}=-\frac{I_{1}^{4}}{\mu^{2}}\left(1+\frac{3}{2}\left(1-\frac{I_{2}^{2}}{I_{1}^{2}}\right)\right)=-\left(\frac{5}{2} \frac{I_{1}^{4}}{\mu^{2}}-\frac{3}{2} \frac{I_{1}^{2} I_{2}^{2}}{\mu^{2}}\right) .
$$

Заменяя здесь $I_{1}$ на $J_{1}$, как это предписывается изложенной процедурой, получаем гамильтониан первого приближения

$$
\begin{aligned}
\mathcal{H} & =H_{0}\left(J_{1}\right)+\varepsilon \bar{H}_{1}\left(J_{1}, I_{2}\right), \\
H_{0}\left(J_{1}\right) & =-\frac{\mu^{2}}{2 J_{1}^{2}}, \quad \bar{H}_{1}\left(J_{1}, I_{2}\right)=-\left(\frac{5}{2} \frac{J_{1}^{4}}{\mu^{2}}-\frac{3}{2} \frac{J_{1}^{2} I_{2}^{2}}{\mu^{2}}\right) .
\end{aligned}
$$

Для изменения угла $\psi_{2}$ в рассматриваемом приближении получаем

$$
\dot{\psi}_{2}=\varepsilon \frac{\partial \bar{H}_{1}\left(J_{1}, I_{2}\right)}{\partial I_{2}}=3 \varepsilon J_{1}^{2} I_{2} / \mu^{2} .
$$

Эта формула дает в рассматриваемом приближении среднюю частоту вращения перицентра. Погрешность этой формулы $\sim \varepsilon^{2}$. Без увеличения погрешности можно считать, что $J_{1}$ связано с величиной энергии $h$ соотношением $h=-\mu^{2} /\left(2 J_{1}^{2}\right)$. Определяя отсюда $J_{1}$ и подставляя в (5.8), получаем для средней частоты вращения перицентра выражение

$$
\dot{\psi}_{2}=\frac{3}{2} \varepsilon \frac{I_{2}}{|h|} .
$$

Для изменения угла $\psi_{1}$ в рассматриваемом приближении получаем

$$
\dot{\psi}_{1}=\frac{\partial H_{0}\left(J_{1}\right)}{\partial J_{1}}+\varepsilon \frac{\partial \bar{H}_{1}\left(J_{1}, I_{2}\right)}{\partial J_{1}},
$$


или, в явном виде

$$
\dot{\psi}_{1}=\frac{\mu^{2}}{J_{1}^{3}}-\frac{\varepsilon}{\mu^{2}}\left(10 J_{1}^{3}-3 J_{1} I_{2}^{2}\right) .
$$

Эта формула дает в рассматриваемом приближении среднюю частоту изменения средней аномалии. Погрешность этой формулы $\sim \varepsilon^{2}$. Без увеличения погрешности можно во втором члене этой формулы определять $J_{1}$ из соотношения $h=-\mu^{2} /\left(2 J_{1}^{2}\right)$, поскольку сам этот член уже порядка $\varepsilon$. В первом члене этой формулы, поскольку он порядка 1, нужно действовать точнее: использовать для определения $J_{1}$ соотношение $\mathcal{H}=h$ и формулы (5.7), т.е.

$$
-\frac{\mu^{2}}{2 J_{1}^{2}}-\frac{\varepsilon}{\mu^{2}}\left(\frac{5}{2} J_{1}^{4}-\frac{3}{2} J_{1}^{2} I_{2}^{2}\right)=h .
$$

Для $J_{1}$ с погрешностью порядка $\varepsilon^{2}$ отсюда получаем

$$
\frac{1}{J_{1}^{3}}=\left(\frac{-2 h}{\mu^{2}}\right)^{3 / 2}-\frac{3 \varepsilon}{2 \mu^{4}}\left[\frac{5}{\left(-2 h / \mu^{2}\right)^{3 / 2}}-\frac{3}{\left(-2 h / \mu^{2}\right)^{1 / 2}} I_{2}^{2}\right]+O\left(\varepsilon^{2}\right) .
$$

Теперь в главном приближении из (5.11) получаем

$$
\dot{\psi}_{1}=\mu^{2}\left(\frac{-2 h}{\mu^{2}}\right)^{3 / 2}-\frac{\varepsilon}{\mu^{2}}\left[\frac{35}{2\left(-2 h / \mu^{2}\right)^{3 / 2}}-\frac{15}{2\left(-2 h / \mu^{2}\right)^{1 / 2}} I_{2}^{2}\right] .
$$

Эта формула определяет среднюю частоту движения тел относительно положения перицентра.

В обозначениях разделов 2, 3 формулы для средней частоты прецессии перицентра $\langle\dot{g}\rangle \equiv \dot{\psi}_{1}$, и средней частоты изменения средней аномалии $\langle\dot{l}\rangle \equiv \dot{\psi}_{2}$ принимают вид (мы выражаем результаты через сохраняющиеся величины: полную энергию $E$ и угловой момент $L)$ :

$$
\begin{aligned}
\langle\dot{g}\rangle & =\frac{\Lambda c^{2}}{4} \frac{L}{|E|}, \\
\langle\dot{l}\rangle & =\frac{(2|E| / m)^{3 / 2}}{G M}-\frac{5 \Lambda c^{2}}{12 G M}\left[\frac{7(G M)^{2}}{(2|E| / m)^{3 / 2}}-\frac{3}{(2|E| / m)^{1 / 2}}(L / m)^{2}\right] .
\end{aligned}
$$

Вид этих формул упрощается, если использовать в них усредненные значения большой полуоси и экцентриситета $\bar{a}, \bar{\epsilon}$, определяемые соотношениями $J_{1}=\sqrt{G M \bar{a}}$, $I_{2}=\sqrt{G M \bar{a}\left(1-\bar{\epsilon}^{2}\right)}$ :

$$
\begin{aligned}
\langle\dot{g}\rangle & =\frac{\Lambda c^{2}}{2 \bar{\omega}} \sqrt{1-\bar{\epsilon}^{2}}, \\
\langle\dot{l}\rangle & =\bar{\omega}-\frac{\Lambda c^{2}}{6 \bar{\omega}}\left(7+3 \bar{\epsilon}^{2}\right) .
\end{aligned}
$$

Здесь $\bar{\omega}=\sqrt{G M} / \bar{a}^{3 / 2}$ - среднее движение для невозмущенной кеплеровской орбиты с большой полуосью $\bar{a}$. Первая из этих формул получена в [22], см. (3.2). 
Величины $\bar{a}, \bar{\epsilon}$ выражаются через полную энергию $E$ и угловой момент $L$ с погрешностью $O\left(\varepsilon^{2}\right)$ по следующим формулам

$$
\begin{aligned}
\bar{a} & =\frac{G M}{2|E| / m}+\frac{\Lambda c^{2} G M}{48(|E| / m)^{3}}\left(5 \frac{(G M)^{2}}{2|E| / m}-3(L / m)^{2}\right) \\
\bar{\epsilon} & =\sqrt{1-\frac{(L / m)^{2}(2|E| / m)}{(G M)^{2}}}+\frac{\Lambda c^{2}(L / m)^{2}}{24}\left(5 \frac{(G M)^{2}}{2|E| / m}-3(L / m)^{2}\right)\left(1-\frac{(L / m)^{2}(2|E| / m)}{(G M)^{2}}\right)^{-1 / 2}
\end{aligned}
$$

Формула (5.16) позволяет найти время между прохождениями через перицентр с учетом возмущения (период орбитального движения). В рассматриваемом приближении это время равно

$$
\begin{aligned}
T & =\frac{2 \pi}{\langle\dot{l}\rangle}=\frac{2 \pi}{\frac{(2|E| / m)^{3 / 2}}{G M}-\frac{5 \Lambda c^{2}}{12 G M}\left[\frac{7(G M)^{2}}{(2|E| / m)^{3 / 2}}-\frac{3}{(2|E| / m)^{1 / 2}}(L / m)^{2}\right]} \\
& \approx \frac{2 \pi G M}{(2|E| / m)^{3 / 2}}+\frac{2 \pi G M}{(2|E| / m)^{3}} \frac{5 \Lambda c^{2}}{12}\left[\frac{7(G M)^{2}}{(2|E| / m)^{3 / 2}}-\frac{3}{(2|E| / m)^{1 / 2}}(L / m)^{2}\right] .
\end{aligned}
$$

Здесь период выражен через первые интегралы исходной задачи. Можно без изменения порядка погрешности выразить член пропорциональный $\Lambda$ через $\bar{a}$ и $\bar{\epsilon}$ :

$$
T=\frac{2 \pi G M}{(2|E| / m)^{3 / 2}}+\frac{5 \pi \Lambda c^{2}}{6 \bar{\omega}^{3}}\left(4+3 \bar{\epsilon}^{2}\right) .
$$

Общая картина движения в рассматриваемой задаче такова. Большая полуось и эксцентриситет кеплеровского эллипса испытывают малые периодические колебания около постоянных значений $\bar{a}, \bar{\epsilon}$; долгота перицентра и средняя аномалия испытывают малые периодические колебания около равномерных вращений с частотами, задаваемыми формулами (5.15), (5.16). Эти малые колебания определяются из соотношений замены переменных (5.1). Ввиду громоздкости формул, мы ограничимся нахождением функции $S$ и колебаний большой полуоси и эксцентриситета.

Согласно (5.2) и (4.2),

$$
S=-\frac{1}{\bar{\omega}} \int\left(H_{1}-\bar{H}_{1}\right)(1-\epsilon \cos \xi) d \xi
$$

Здесь $\bar{\omega}=\partial H_{0}\left(J_{1}\right) / \partial J_{1}$ - частота кеплеровского движения, посчитанная в главном приближении. Использование в записи $S$ неопределенного интеграла указывает, что $S$ определена с точностью до произвольной функции от медленных переменных. В дальнейшем мы выберем эту произвольную функцию так, чтобы среднее от $S$ по $\varphi_{1}$ равнялось 0. Учитывая, 
что $r=a(1-\epsilon \cos \xi)$, и используя (5.4), получаем

$$
\begin{aligned}
S & =\frac{1}{\bar{\omega}}\left[\left(\int a^{2}(1-\epsilon \cos \xi)^{3} d \xi\right)-a^{2}\left(1+\frac{3 \epsilon^{2}}{2}\right)(\xi-\epsilon \sin \xi)\right] \\
& =\frac{a^{2}}{\bar{\omega}}\left[\left(\int\left(1-3 \epsilon \cos \xi+3 \epsilon^{2} \frac{1+\cos 2 \xi}{2}-\epsilon^{3} \frac{\cos 3 \xi+3 \cos \xi}{4}\right) d \xi\right)-\left(1+\frac{3 \epsilon^{2}}{2}\right)(\xi-\epsilon \sin \xi)\right] \\
& =\frac{a^{2}}{\bar{\omega}}\left[-3 \epsilon \sin \xi+\frac{3}{4} \epsilon^{2} \sin 2 \xi-\frac{1}{12} \epsilon^{3}(\sin 3 \xi+9 \sin \xi)+\epsilon\left(1+\frac{3 \epsilon^{2}}{2}\right) \sin \xi\right]+C \\
& =\frac{a^{2}}{\bar{\omega}}\left[\epsilon\left(-2+\frac{3}{4} \epsilon^{2}\right) \sin \xi+\frac{3}{4} \epsilon^{2} \sin 2 \xi-\frac{1}{12} \epsilon^{3} \sin 3 \xi\right]+C .
\end{aligned}
$$

Здесь $C$ - произвольная функция от $a, \epsilon$. Мы выберем $C$ так, чтобы среднее от $S$ по $\varphi_{1}$ равнялось 0. Получаем

$$
C=-\frac{a^{2}}{\bar{\omega}} \int_{0}^{2 \pi}\left(\epsilon\left(-2+\frac{3}{4} \epsilon^{2}\right) \sin \xi+\frac{3}{4} \epsilon^{2} \sin 2 \xi-\frac{1}{12} \epsilon^{3} \sin 3 \xi\right)(1-\epsilon \cos \xi) d \xi=0 .
$$

Окончательно

$$
S=\frac{a^{2}}{\bar{\omega}}\left[\epsilon\left(-2+\frac{3}{4} \epsilon^{2}\right) \sin \xi+\frac{3}{4} \epsilon^{2} \sin 2 \xi-\frac{1}{12} \epsilon^{3} \sin 3 \xi\right]
$$

где $a, \epsilon$ должны быть заменены на $\bar{a}, \bar{\epsilon}$ и выражены через $J_{1}, I_{2}$ согласно (5.5), а эксцентрическая аномалия $\xi$ должна быть выражена через среднюю аномалию $\varphi_{1}$ согласно (4.2).

Согласно (5.1) и (5.2), величины $I_{1}$ и $J_{1}$ связаны соотношением

$$
I_{1}=J_{1}-\varepsilon \frac{1}{\bar{\omega}}\left(H_{1}-\bar{H}_{1}\right)
$$

Используя (5.4), получаем

$$
\left.I_{1}=J_{1}+\varepsilon \frac{\bar{a}^{2}}{\bar{\omega}}\left((1-\bar{\epsilon} \cos \xi)^{2}-\left(1+\frac{3 \bar{\epsilon}^{2}}{2}\right)\right)=J_{1}+\varepsilon \frac{\bar{a}^{2}}{\bar{\omega}}\left(-\bar{\epsilon}^{2}-2 \bar{\epsilon} \cos \xi+\frac{1}{2} \bar{\epsilon}^{2} \cos 2 \xi\right)\right) .
$$

Согласно (4.2) $I_{1}=\sqrt{G M a}, J_{1}=\sqrt{G M \bar{a}}$. Тогда

$$
\left.\sqrt{G M a}=\sqrt{G M \bar{a}}+\varepsilon \frac{\bar{a}^{2}}{\bar{\omega}}\left(-\bar{\epsilon}^{2}-2 \bar{\epsilon} \cos \xi+\frac{1}{2} \bar{\epsilon}^{2} \cos 2 \xi\right)\right)
$$

откуда, в рассматриваемом приближении,

$$
\left.a=\bar{a}+\varepsilon \frac{2}{\sqrt{G M}} \frac{\bar{a}^{5 / 2}}{\bar{\omega}}\left(-\bar{\epsilon}^{2}-2 \bar{\epsilon} \cos \xi+\frac{1}{2} \bar{\epsilon}^{2} \cos 2 \xi\right)\right) .
$$

Учитывая, что $\bar{\omega}=\sqrt{G M} / \bar{a}^{3 / 2}$, а также что $\varepsilon=\Lambda c^{2} / 6$, получаем

$$
a=\bar{a}+\frac{\Lambda c^{2} \bar{a}}{3 \bar{\omega}^{2}}\left(-\bar{\epsilon}^{2}-2 \bar{\epsilon} \cos \xi+\frac{1}{2} \bar{\epsilon}^{2} \cos 2 \xi\right) .
$$


Сохранения углового момента позволяет определить колебания эксцентриситета по колебаниям большой полуоси. Из соотношения $a\left(1-\epsilon^{2}\right)=\bar{a}\left(1-\bar{\epsilon}^{2}\right)$ получаем

$$
\epsilon=\bar{\epsilon}+\frac{\Lambda c^{2}\left(1-\bar{\epsilon}^{2}\right)}{6 \bar{\omega}^{2}}\left(-\bar{\epsilon}-2 \cos \xi+\frac{1}{2} \bar{\epsilon} \cos 2 \xi\right) .
$$

Введем углы $\bar{l}=\psi_{1}, \bar{g}=\psi_{2}$. Эти углы изменяются равномерно с угловыми скоростями (5.18), (5.17). Средняя аномалия $l=\varphi_{1}$ и долгота перицентра $g=\varphi_{2}$ совершают малые колебания относительно этих углов в соответствии с двумя последними формулами в (5.1), которые без изменения погрешности представляются в виде

$$
l=\bar{l}-\varepsilon \frac{\partial S}{\partial I_{1}}, g=\bar{g}-\varepsilon \frac{\partial S}{\partial I_{2}} .
$$

Здесь функция $S=S\left(I_{1}, I_{2}, \bar{l}\right)$ задается формулой $(5.23)$, в которой большая полуось орбиты $a$ и экцентриситет $\epsilon$ должны быть выражены через переменные Делоне $I_{1}, I_{2}$ из соотношений (4.2), а эксцентрическая аномалия $\xi$ рассматривается как неявная функция от $\bar{l}$ согласно уравнению Кеплера $\bar{l}=\xi-\epsilon \sin \xi$.

\section{6 Заключение}

Задача о движении двух гравитирующих тел при наличии ТЭ рассмотрена как возмущение задачи Кеплера. В рамках первого приближения в теории возмущений ДелонеЦейпеля описано поведение элементов кеплеровской орбиты под влиянием ТЭ. Большая полуось и эксцентриситет кеплеровской орбиты испытывают малые периодические колебания около своих средних значений. Эти средние значения даются формулами (5.19), а колебания - формулами (5.25) и (5.26). Долгота перицентра испытывает малые периодические колебания относительно медленного равномерного вращения с частотой (5.17). Ранее формула для этой частоты была получена несколько иным методом в [22]. Средняя аномалия гравитирующих тел испытывает малые периодические колебания относительно медленного равномерного вращения с частотой, даваемой формулой (5.18). Эта формула учитывает поправку к среднему движению за счет ТЭ. Колебания относительно этих равномерных вращений даются формулами (5.27) (мы не проводим в явном виде вычислений в (5.27) ввиду их громоздкости). Период орбитального движения, определяемый как время между прохождениями через перицентр, отличается от кеплеровского периода малой поправкой. Эта поправка учтена в формуле для периода (5.21). Колебания элементов орбиты происходят с этим периодом.

Работа ГСБК частично поддержана грантами РФФИ №o 18-02-00619, 20-02-000455, 20-52-12053. Работа АИН частично поддержана грантом фонда Леверхульме № RPG-2018143. 


\section{Список литературы}

[1] S. Perlmutter et al., Astrophys. J. 517, 565 (1999).

[2] A.G. Riess et al., Astron. J. 116, 1009 (1998).

[3] D.N. Spergel et al., Astrophys. J. Suppl. 148, 175 (2003).

[4] M. Tegmark et al., Phys. Rev. D 69, 103501 (2004).

[5] S. Weinberg, Rev. Mod. Phys. 61, 1 (1989).

[6] S.M. Carroll, W.H. Press and E.L. Turner, Ann. Rev. Astron. Astrophys. 30, 499 (1992).

[7] S. M. Carroll, Living Rev. Relativ. 4, 1 (2001).

[8] W. Rindler, Relativity: Special, General, and Cosmological (Oxford University Press, Oxford, UK, 2001).

[9] C. O'Raifeartaigh, M. O'Keeffe, W. Nahm and S. Mitton, Eur. Phys. J. H 43, 73 (2018).

[10] B. Novosyadlyj, Eur. Phys. J. H 43, 267 (2018).

[11] L. Iorio, Universe 1, 38 (2015).

[12] I. Debono and G. F. Smoot, Universe 2, 23 (2016).

[13] J. Islam, Phys. Lett. A 97, 239 (1983).

[14] J. Cardona and J. Tejero, Astrophys. J. 493, 52 (1998).

[15] L. Iorio, Int. J. Mod. Phys. D 15, 473 (2006).

[16] L. Iorio, Advances in Astronomy 2008, Article ID 268647 (2008).

[17] H. Arakida, Int. J. Theor. Phys. 52, 1408 (2013).

[18] S.S. Ovcherenko and Z.K. Silagadze, Ukr. J. Phys. 61, 342 (2016).

[19] L. Iorio, Universe 4, 59 (2018).

[20] J.M. Cohen and B. Mashhoon, Phys. Lett. A 181, 353 (1993).

[21] E. Hackmann, C. Lammerzahl Phys. Rev. D 90, 044059 (2014).

[22] A. Kerr, J. Hauck and B. Mashhoon, Class. Quantum Grav. 20, 2727 (2003). 
[23] P. Jetzer and M. Sereno, Phys. Rev. D 73, 044015 (2006).

[24] M. Sereno and P. Jetzer, Phys. Rev. D 73, 063004 (2006).

[25] V. Kagramanova, J. Kunz and C. Lammerzahl, Phys. Lett. B 634, 465 (2006).

[26] G. Adkins, J. McDonnell and R. Fell, Phys. Rev. D 75, 064011 (2007).

[27] G. Adkins and J. McDonnell, Phys. Rev. D 75, 082001 (2007).

[28] M. Sereno and P. Jetzer, Phys. Rev. D 75064031 (2007).

[29] O.I. Chashchina and Z.K. Silagadze, Phys. Rev. D 77, 107502 (2008).

[30] A.D. Chernin, Phys. Usp. 44, 1099 (2001).

[31] A.D. Chernin, Phys. Usp. 51, 253 (2008).

[32] G.S. Bisnovatyi-Kogan and A.D. Chernin, Astrophysics and Space Science, 338, 337 (2012).

[33] G.S. Bisnovatyi-Kogan, M. Merafina, Int. J. Mod. Phys. D28, 1950155 (2019)

[34] N.V. Emelyanov and M.Y. Kovalyov, Mon. Not. R. Astron. Soc. 429, 3477 (2013).

[35] I. S. Gradshteyn, I. M. Ryzhik, Table of integrals, series and products (1965) New York: Academic Press, edited by Geronimus, Yu.V., Tseytlin, M.Yu. (4th ed.).

[36] L. Landau, E. Lifshitz, Mechanics. Course of theoretical physics (1969) Pergamon Press.

[37] Г.Н.Дубошин, Справочное руководство по небесной механике и астродинамике (1976) Наука.

[38] Г.Е.О.Джакалья, Методы теории возмущений для нелинейных систем (1979) Наука. 